\title{
ALPS evaluation in Financial Portfolio Optimisation
}

\author{
S. Patel and C. D. Clack
}

\begin{abstract}
Hornby's Age-Layered Population Structure claims to reduce premature convergence in Evolutionary Algorithms. We provide the first evaluation of ALPS on a realworld problem - the evolution of non-linear factor models for financial portfolio optimisation. We incorporate ALPS into our GP system, coupled to an investment simulator, and provide a head-to-head comparison between ALPS GP and Standard GP. By investigating the performance of ALPS both during training and during out-of-sample validation, we provide empirical evidence of the benefits of ALPS; we show that it really does reduce convergence, and provides fitter individuals, in our problem domain. The ALPS GP system evolves non-linear factor models that out-perform not only the Standard GP system, but also the market index by a significant amount.
\end{abstract}

\section{INTRODUCTION}

Hornby has introduced the Age-Layered Population Structure (ALPS) [6], a new system to reduce the problem of premature convergence in evolutionary algorithms. ALPS uses a novel measure for an individual's age, based on the amount of time the individual's genotypic material has been evolving in the population, and splits the population into separate layers each of which contains individuals whose ages lie in a particular range. Breeding is restricted to individuals that occupy the same age layer.

We have extensively tested ALPS on a real-world problem by incorporating it into a Genetic Programming (GP) system and provide empirical evidence to demonstrate the value of the technique.

In our research into the applicability of Genetic Programming (GP) technology to the optimization of investment portfolios, we have constructed an automated investment simulator that uses GP to derive a profitable non-linear relationship between a large number of factors relating to equities. This non-linear factor model assists the system in making buy and sell decisions. Our system has been trained on a basket of 82 FTSE100 stocks, and displays successful investment behaviour in validation tests (the FTSE100 is an index of the $100 \mathrm{UK}$ equities with greatest capitalisation). However, standard GP systems are characterized by the fact that the final trained population often has little diversity and has converged very early in training. The individuals in the final generation therefore tend to be less fit than they should be (i.e. they represent, or approximate, a local optimum rather than the global optimum), and less general (i.e. they tend to be more over-fitted to the training data).

By incorporating ALPS into our GP system and investment simulator we aim to determine:

The authors are with the Department of Computer Science, University College London, Gower Street, London WC1E 6BT (email: clack@cs.ucl.ac.uk)
- the efficacy of the new ALPS incorporated system i.e. to what extent it reduces premature convergence of the fittest individual, compared with a standard GP system;

- the impact of ALPS on the amount of over-fitting in the GP population, determined by the results of testing on out-of-sample data; and

- the overall best fitness attained by the population during training and validation, compared with a standard GP system.

\section{RELATED WORK}

Many techniques for the reduction of premature convergence and the preservation of diversity exist. For example, the Hierarchical Fair Competition (HFC) model [7] is very similar to ALPS. It splits the population into layers and also introduces randomly generated individuals into the bottom layer. However HFC uses fitness rather than age to segregate individuals into layers. This results in the problem of individuals that have converged to a local optima near the top fitness layer preventing newer individuals in different basins of attraction from climbing through that fitness layer [6].

The multipopulation genetic programming [17] technique also uses a method based on the segregation of individuals. However, the system differs from ALPS and HFC as it involves an initial population split into subpopulations. The subpopulations evolve using differing mutation and crossover probabilities. Each subpopulation can communicate and transfer their best individuals every few generations. However, an individual can only move into a subpopulation with a lower fitness than its own. This would lead to a reduction in diversity given the newly migrated individual will have a higher fitness than the rest of the subpopulation and dominate the subpopulation during crossover and mutation. Therefore the rate at which individuals are allowed to move into new subpopulations must be carefully considered. There is also no introduction of randomly generated individuals and there is no guarantee that all subpopulations will not converge to the same local optimum.

Diversity preservation techniques such as the Adaptive Diversity Maintenance [2] technique aim to increase diversity preservation at specific points in the evolutionary run and at others aim to gain from convergence. The main structure is to ensure diversity preservation at the start of the run and to transfer focus to convergence towards the end of the run to get higher fitnesss. However this method does not enable the introduction of randomly generated individuals and so in any state either convergence or diversity preservation individuals are around the same basin of attraction and only the speed 
at which they reach their local optima is controlled, but not the actual diversity of the population.

\section{A. GP in portfolio optimisation}

The notion that stock market prices follow a random walk was first introduced by Kendall in 1953 [11] and has subsequently been promoted by Malkiel in his book "A Random Walk Down Wall Street" [13]. Malkiel was also a major proponent of Fama's Efficient Market Hypothesis(EMH)[3]. According to the EMH, future price predictions cannot be made on the basis of past movements in price. The strong version of the EMH goes further to state that no amount of past technical or fundamental data analysis will allow the prediction of future price movements.

The EMH however is no longer as widely respected as it once was, and it is no longer heresy to argue firstly that inefficiencies in markets do occur, especially where trading volumes are low, and secondly that these inefficiencies can be exploited profitably. See, for example [12].

There have been many applications of evolutionary algorithms to the problems of price prediction, trading, and portfolio optimisation; those that specifically use Genetic Algorithm (GA) and GP techniques include [9], [10], [15], [16], [18], [19], [20]. Adaptive trading systems have also been explored for the FX market by Dempster [1], in which trading decisions are made based on a continuously changing set of trading rules based on real-time market conditions. Further GP applications to finance include work by Iba and Sasaki [8], in using GP to predict the price of the Nikkei index, and similarly the application of GP to the price movement of the NASDAQ and the S\&P CNX by Grosan and Abraham [5].

Systems that attempt to beat the market based solely on price prediction on a daily basis still have some way to go to successfully circumvent the volatility displayed in the world's stock markets. By contrast, prediction of stock value and medium-term future value based on solid fundamental analysis of stocks and their markets is a well researched area in Finance (see for example [4]).

Our system differs to much of the prior work in this area because we do not attempt to predict the movement of prices on a daily basis, nor do we use purely technical data. Rather, we make predictions of the best stock to buy based on a large number of both technical and fundamental factors related to that stock. Thus, our system is not influenced purely by price movements but also by the underlying fundamental commercial performance of the companies whose stocks we monitor - see for example [4] for an explanation of fundamental analysis.

\section{INTRODUCTION TO ALPS}

The ALPS technique introduces a novel way of measuring an individual's age and segregating individuals into agelayers. Age is measured by the number of generations an individual's genotypic material has been evolving rather than the individual itself. This method of measuring age is in contrast to many other age-based systems, as new individuals created by mutation or recombination take the age of their eldest parent plus 1 rather than starting at 0 . By contrast, new randomly generated individuals start with an age of 0 as their genotypic material has not yet been through the evolution process.

An individual's age is incremented if it is used as a parent in breeding and is copied to the next generation through elitism. The age of a individual is only incremented by 1 each generation irrespective of how many children it creates in that generation. If the individual is not used as a parent its age will remain unchanged.

Individuals are held within age layers which are defined by a given age gap that dictates the range of ages held within each layer. The age layers restrict competition and breeding to within each layer and ensure younger individuals have a number of generations to find and move into a good basin of attraction before they are pushed into the next layer.

ALPS imposes numerous restrictions on the evolution process to ensure control of the breeding process, maintain diversity and reduce the problem of premature convergence.

- An individual can only breed with another individual either within its own layer or within the previous layer.

- At regular intervals all individuals in the bottom age layer are replaced with randomly generated individuals.

- An age layer is created only when the current generation is equal to the age limit for the previous layer. For example with an age gap of 15 a new age layer will be created at generation 0 , layer 2 will be created at generation 15 , layer 3 at generation 30 , layer 4 at generation 45 , etc.

New layers are populated by those individuals that have surpassed the previous layer's age limit. This will usually be the offspring of individuals from the previous layer. Subsequent movement occurs only if an individual is now too old for its layer and its fitness is better than an individual in the next layer. If the individual is not better than one in the next layer it is removed from the population. It does not go back to its current age layer as it is too old for that layer. An individual is only guaranteed to stay in the population forever if it is at the global optimum; otherwise it will eventually be replaced as better individuals are evolved or it is unable to move into the next layer when it is too old [6].

\section{DESCRIPTION OF THE System}

To test the efficacy of the ALPS technique against the standard GP, we utilise our simulator of a long-only trading investment portfolio of FTSE stocks. The GP system evolves a non-linear equation that uses market data to determine whether each stock should be selected to buy. Thus, each individual is a tree comprising: (i) terminals, that are either constants or identifiers relating to technical or fundamental data for the stock, and (ii) non-terminals, that are standard operators such as,$+ \times,-, \div, \sqrt{ }$ etc. An example individual is illustrated in Figure 1.

Creating the ALPS GP required a substantial overhaul of the breeding process of the standard GP; however both 


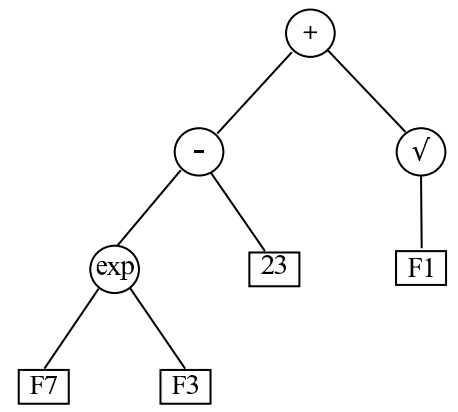

Fig. 1. Sample individual in the GP population.

systems use the same investment simulator subsystem. The coupling between the GP and Investment Simulator subsystems is the fitness function - the investment simulator is called each time the GP subsystem needs to determine the fitness of an individual, at which point the individual is used to control the simulation of an investment portfolio of FTSE stocks. The simulator uses an algorithm supplied by an industry partner; this is applied to training data of monthly prices and other technical and fundamental factors for a period of 30 months. Monthly returns on investment are calculated and at the end of the 30 months simulation the Sharpe Ratio (a standard metric used by investment managers [14]) is calculated and returned to the GP system as the individual's fitness.

The Sharpe Ratio is given in Equation 1, where $S$ is the Sharpe Ratio over the training period, $\bar{x}$ is the average monthly Return On Investment (ROI), $\sigma$ is the standard deviation of monthly ROIs, and $R F R$ is the average monthly Risk Free Rate. We set $R F R$ to 0.003 (equivalent to $3.6 \%$ per annum).

$$
S=\frac{\bar{x}-R F R}{\sigma}
$$

Fund managers often set a target Sharpe Ratio, as do we - our target is 1.5 , and we utilise an adjusted fitness that is given by the absolute difference between the measured Sharpe Ratio and the target.

\section{The InVESTMENT Simulator}

We simulated a long only trading strategy for FTSE 100 stocks. The training data consisted of 30 months of technical and fundamental data including monthly prices. We traded a portfolio of 20 stocks on a monthly basis and all trading took place at the start of each month and the portfolio held for the duration of the month. At the beginning of each month we used the individual provided by the GP system as a stock selection model that quantitatively measures the attractiveness of each stock; this model was a non-linear combination of technical and fundamental factors to predict the return expectation for each stock over a 4 week forward horizon. Each stock was ranked according to the expected return and then the top 20 were bought and held in the

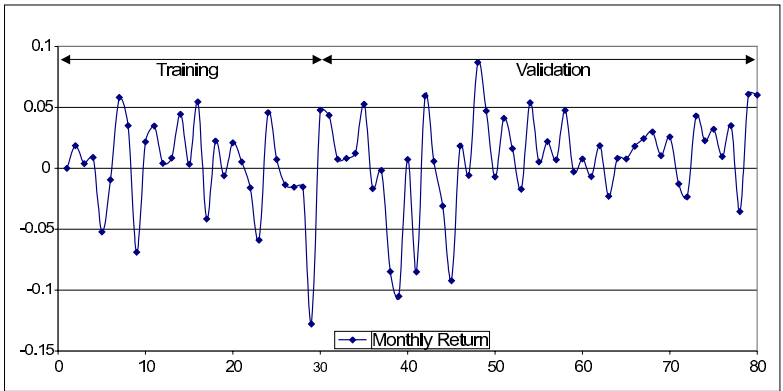

Fig. 2. Market index fractional monthly returns (80 months: 31 st May 1999 to 31st December 2005)

portfolio for that month. Positions are closed at the end of each month and an investment of $£ 1,000,000$ takes place each month regardless of the profit or loss in the preceding month. The process takes place through the 30 months of data for each individual in the population.

\section{EXPERIMENT}

Our primary research question is: "Does ALPS reduce premature convergence and provide individuals that are fitter both during training and during validation?"

Our experiment will compare the performance of the standard GP against the ALPS GP. We also compare performance of both systems against a market index.

\section{A. Data}

Both systems use an investment simulator that has an investment universe of 82 FTSE 100 stocks. The training data for both systems comprises time-series financial data for the 82 stocks taken from the period 31st May 1999 to 31st October 2001. The data is primarily provided by Reuters, and comprises both technical and fundamental data. A list of example factors is given in Table I.

TABLE I

EXAMPLE FACTORS USED AS TERMINALS FOR GP INDIVIDUALS

\begin{tabular}{|l|l|}
\hline Price & The average stock price this month \\
Price Momentum & The acceleration of price this month \\
Volume & Number of shares traded this month \\
Price/Earnings Ratio & Capitalisation $\div$ annual earnings \\
30d Moving Average & 30 days MA of the price \\
Volatility & Standard deviation of stock prices this month \\
Dividend Yield & Dividend per share $\div$ share price \\
BVPS & Book Value of the company $\div$ issued shares \\
Capitalisation & Current share price $\times$ issued shares \\
EPS & Company earnings $\div$ issued shares \\
Earnings & Earnings in the last financial year \\
Altman Z-Factor & The probability of a company's failure \\
\hline
\end{tabular}

\section{B. The Index}

Although our stocks are selected from the FTSE100 constituents, we do not compare performance against the FTSE100 index (since we might have unwittingly selected only stocks that perform well) — instead, we construct 


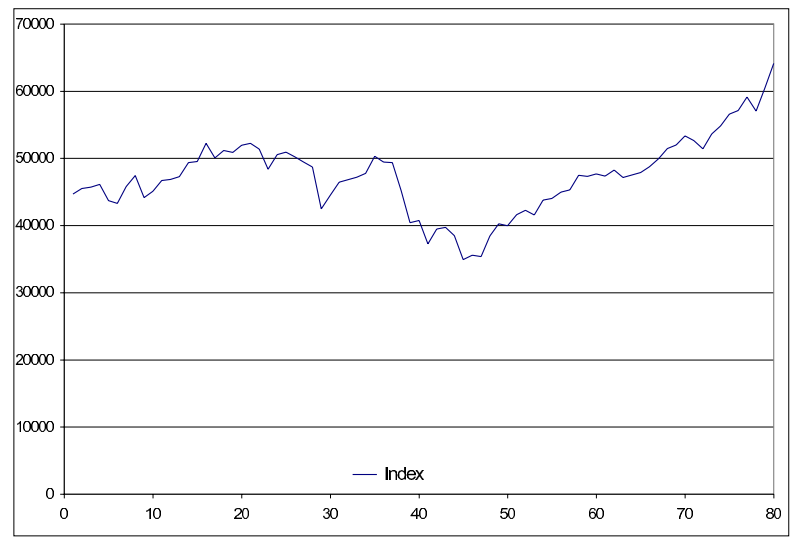

Fig. 3. Market Index Value (80 months: 31st May 1999 to 31st December 2005)

an unweighted index comprising just those stocks that are available to our simulator. We call this our "market index".

Figure 2 illustrates the monthly percentage change of the market index for our complete universe of 82 stocks during the training and validation periods. The index displays a good mix of volatile, bear and bull periods for training and validation. Figure 2 also indicates that we use the first 30 months for training and the last 50 months for validation. For both training and validation we make 5 runs of the system.

Figure 3 displays the un-weighted sum of the value of all the stocks used in the Investment Simulator each month.

\section{Traded Index}

In order to provide a fair comparison between the performance of the market index, ALPS and the Standard GP, we must simulate the performance of an un-weighted tradable index with the same investment rules as those used in the Investment Simulator.

Figure 4 illustrates the Cumulative return of the index both with and without the trading costs which are applied to the Investment Simulator. The index is bought at the start of each month with $£ 1,000,000$ and the return is calculated by multiplying $£ 1,000,000$ by the percentage change in the un-weighted market index (as shown in Figure 2). Just as with the investment simulator, the investment of $£ 1,000,000$ takes place each month regardless of any profit or loss in the previous month. Our comparison will use the Traded Index without the effects of trading costs (a tougher test for GP!).

\section{Out of Sample Validation}

Both systems are validated on a previously unseen "out of sample" data set, comprising time-series financial data for the 82 stocks taken from the period 31st October 2001 to 31st December 2005. During this period the FTSE suffered a bear market between months 35 and 48 , and towards the end there is an indication of a bull market.

This varied and hostile period provides a real test of the robustness of individuals as they must survive the sharp dip

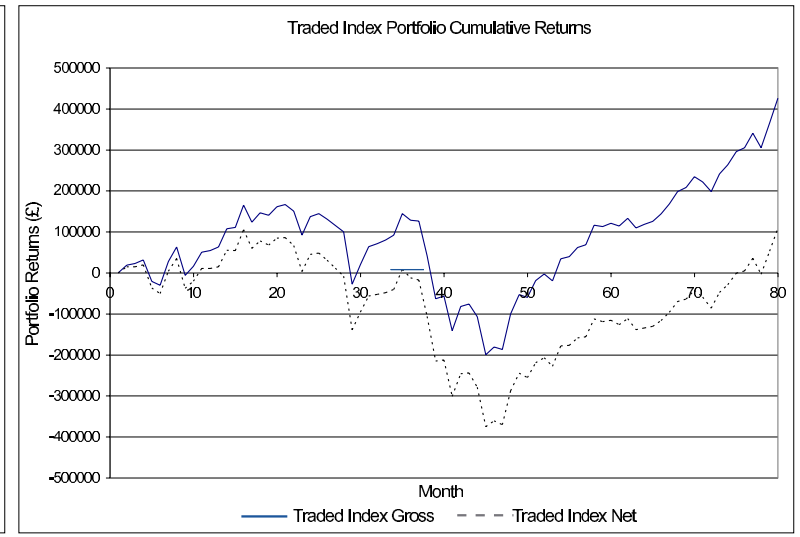

Fig. 4. Traded Index Cumulative Returns; Gross and Net of trading costs.

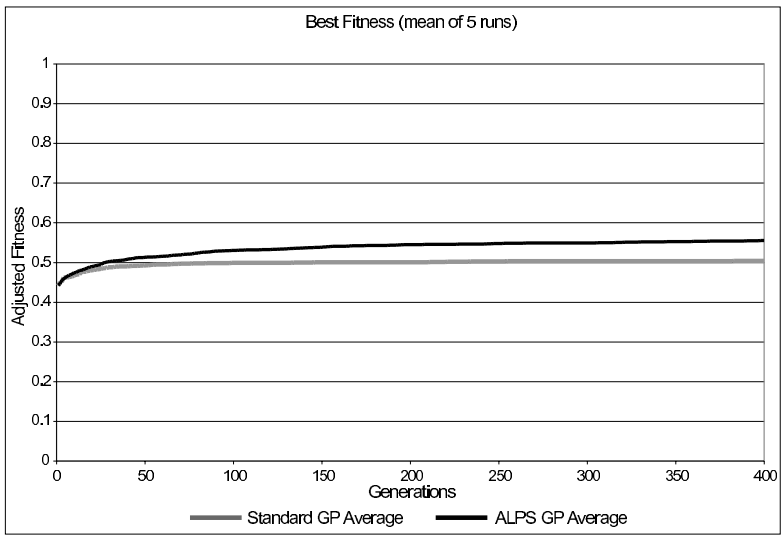

Fig. 5. Best fitness per generation during training.

and maximise available returns during the bull market to beat the benchmark.

We have conducted 5 runs of training for each system and run the validation using 25 individuals from the final populations of the 5 training runs ( 5 from each run). Our statistical analysis therefore compares two populations each with 25 observations.

\section{MAIN RESUltS}

The research question we proposed to answer was "Does ALPS reduce premature convergence and provide individuals that are fitter both during training and during validation?"

Figure 5 illustrates the best fitness of each generation; here we can see that the implementation of ALPS provides a substantial improvement over the standard GP in the reduction of premature convergence. The convergence properties of Standard GP and ALPS GP are compared by defining convergence as follows: the generation beyond which the difference in the best fitness from one generation to the next never again equals or exceeds $1 \%$. Using this 


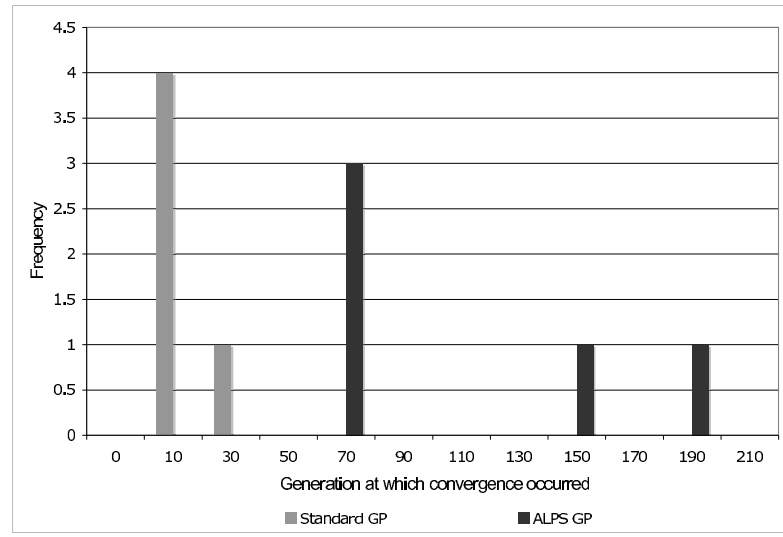

Fig. 6. Convergence behaviour of the two systems.

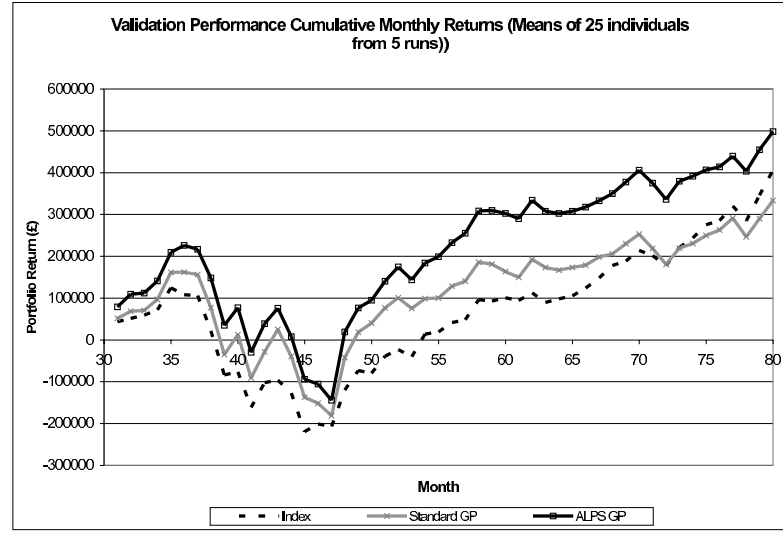

Fig. 7. Cumulative Returns during out-of-sample validation testing

definition, we compare multiple runs of the two systems and plot a histogram of the generations when convergence occurs - a simple example is provided in Figure 6. A Ttest comparison provides a p-value of $1.05 \times 10^{-3}$ which indicates a significant difference in convergence behaviour.

The implementation of ALPS also provides us with the benefit of individuals with considerably higher fitness in the final generation. However the success of individuals during training must be transferable to a unseen environments and so our GP must be able to produce individuals robust enough to succeed when tested on our out-of-sample validation data.

Figure 7 displays the average cumulative returns gained from trading a portfolio of 20 stocks during the validation

TABLE II

AVERAGE PERCENTAGE RETURN FROM VALIDATION PERIOD

\begin{tabular}{|l|c|}
\hline System & Percentage Return \\
\hline ALPS GP & $50 \%$ \\
\hline Index & $40 \%$ \\
\hline Standard GP & $33 \%$ \\
\hline
\end{tabular}

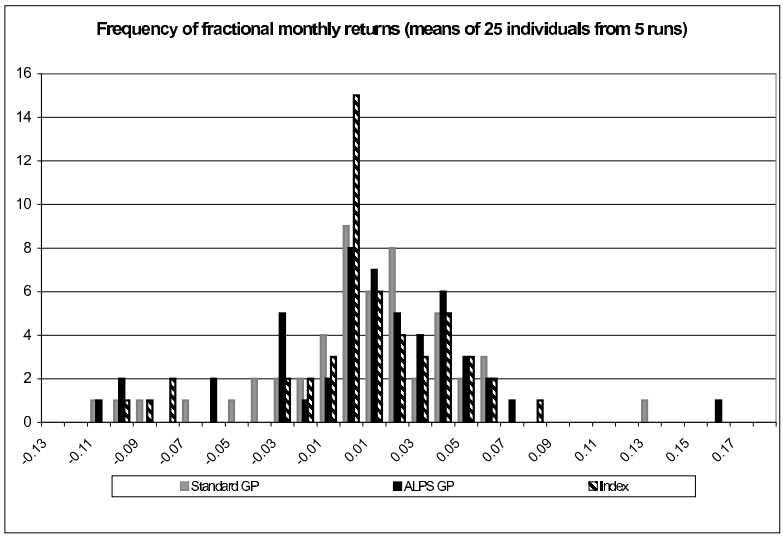

Fig. 8. Frequency distribution of mean monthly fractional returns

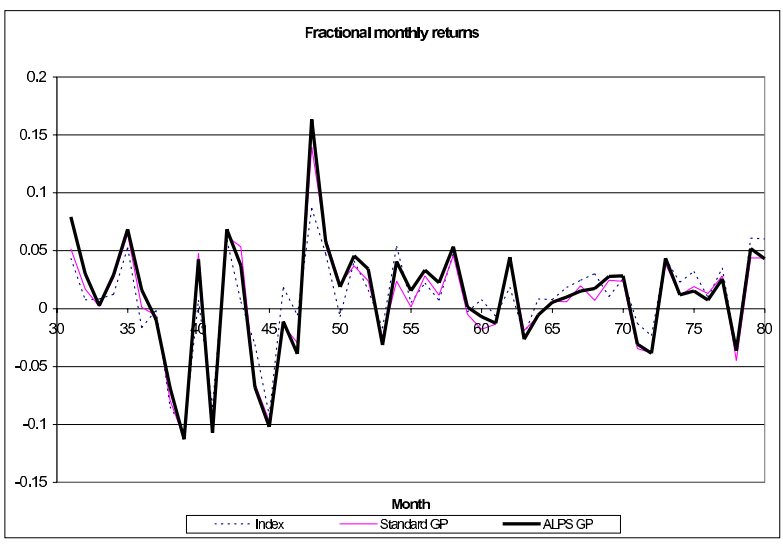

Fig. 9. Fractional Monthly Returns during out-of-sample validation testing

period of 50 months with $£ 1,000,000$ available each month to invest. The index portfolio is made of an un-weighted index of the 82 stocks used in the investment simulation.

The performance of individuals from the ALPS GP not only surpasses that of the Standard GP but beats the benchmark index (by $10 \%$ over the period). The ALPS individual beat the market throughout the entire period, surviving the bear market and successfully riding the bull. We see that although the standard GP is able to beat the index throughout most of the period, it is unable to successfully capitalise on the bull market towards the end of the period. The average percentage return from the validation period, for the two GP systems and the index, are given in Table II.

Figure 8 illustrates the distribution of the average monthly returns from the 25 individuals, for the two GP systems and the index. The volatility of returns in both the systems and the index can be clearly seen in Figure 9. Although we generally view high volatility of returns as undesirable, in the period given the index clearly shows a similar degree 


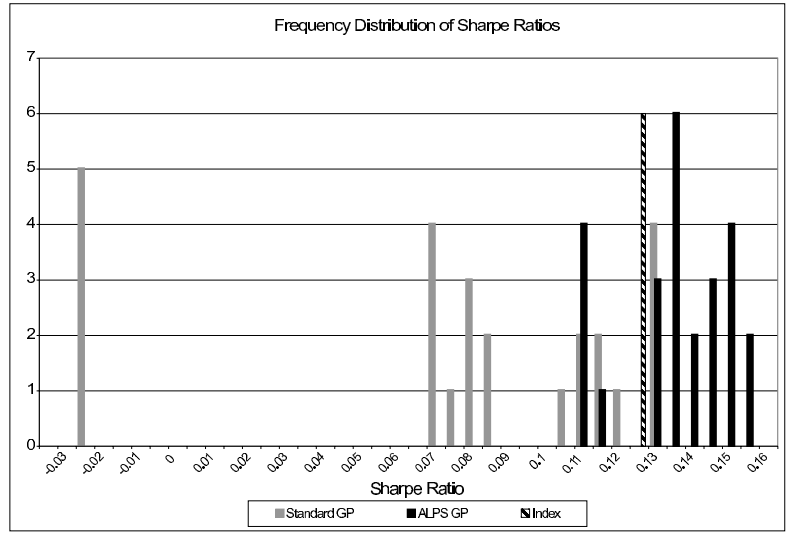

Fig. 10. Frequency distribution of the Sharpe Ratio

TABLE III

RANKED T-TEST RESULTS - COMPARISON OF SHARPE RATIOS

\begin{tabular}{|c|c|}
\hline Comparison & P-Value \\
\hline Standard GP to ALPS GP & $1.77 \times 10^{-10}$ \\
\hline
\end{tabular}

of volatility in the downside at least, therefore the level of volatility is acceptable as long as the returns attained are proportionate to the level of risk. An effective measure for the relation between the average return on investment and the standard deviation or volatility of those returns is the Sharpe Ratio [14]. The Sharpe Ratio is used within the investment simulator to ascertain an individual's fitness but we can use it here to measure the effectiveness in validation of the individuals produced by the Standard GP and the ALPS GP.

Figure 10 illustrates the distribution of Sharpe Ratios after validation tests of the 25 individuals from each system (the Sharpe Ratio of the index, which does not change, is also shown). We can clearly see the disparity between the distributions of the two systems where ALPS provides much higher Sharpe Ratios - that is, ALPS provides a higher return on investment per unit of risk. To accurately measure the disparity and ensure a significant difference we applied a ranked T-test to the data from Figure 10. The results displayed in Table III indicate a convincing difference between the Standard GP and ALPS GP, establishing a strong benefit of ALPS over the Standard GP.

A further analysis into the variance of the performance of the 25 individuals from each system can be made through the distribution of final account balances of each of the 25 individuals from each system. The distribution is shown in Figure 11 and the results of the ranked T-test upon the distribution are shown in Table IV. We can see an even greater significant difference than that of the Sharpe ratio, concluding that the effectiveness of ALPS in training has transferred reliably and with low variance into results during validation.

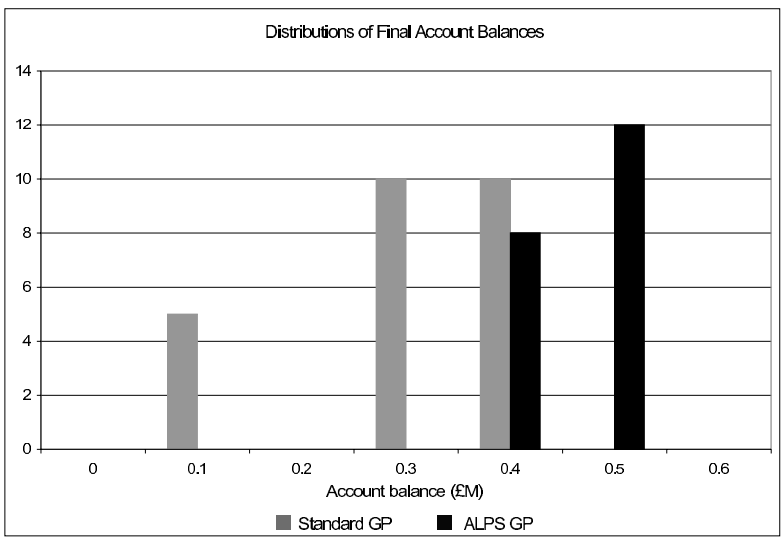

Fig. 11. Distribution of final account balances (validation of 25 individuals from 5 runs of each system

\section{TABLE IV}

RANKED T-TEST RESULTS - COMPARISON OF FINAL PORTFOLIO ACCOUNT BALANCES (OF 25 INDIVIDUALS DRAWN FROM 5 RUNS OF EACH SYSTEM)

\begin{tabular}{|c|c|}
\hline Comparison & P-Value \\
\hline Standard GP to ALPS GP & $2.58894 \times 10^{-15}$ \\
\hline
\end{tabular}

\section{DISCUSSION AND CONCLUSIONS}

Standard GP systems are often characterized by the fact that the final trained population may have little diversity and has converged very early in training. The individuals in the final generation therefore tend to be less fit than they should be (i.e. they represent, or approximate, a local optimum rather than the global optimum), and less general (i.e. they tend to be more over-fitted to the training data).

In the context of our chosen application domain financial portfolio-optimisation - we decided to evaluate Hornby's new ALPS technique that promised to delay convergence and provide fitter individuals in the final population.

We have therefore compared a Standard GP to one incorporated with the ALPS technique, to investigate the benefits of the system in our real world problem. Our research is based on using a GP system for the creation of a nonlinear factor model for stock picking which coupled with an Investment Simulator traded with 82 FTSE 100 stocks over the period 31st May 1999 to 31st December 2005.

Our criteria to determine whether ALPS GP is an improvement to a standard GP covered the following:

- the efficacy of the new ALPS incorporated system i.e. to what extent it reduces premature convergence of the fittest individual, compared with standard GP;

- the impact of ALPS on the amount of over-fitting in the GP population, determined by the results of testing on out-of-sample data; and

- the overall best fitness attained by the population during training and validation, compared with standard GP. 
Our results have shown that in all three cases ALPS GP has surpassed the Standard GP: there is a high statistical difference in the generation at which convergence occurs; a very high statistical difference in the Sharpe Ratio during validation tests; consistently higher fitness results during training; and a very high statistical difference in final portfolio account balances during validation tests.

These are of course preliminary results on a single problem, and we are currently conducting larger-scale experiments on financial data from different periods and different markets. However, the reported T-test results are sufficiently convincing that, in the context of this particular real-world application, we confidently recommend ALPS as an effective optimisation technique.

Further work in the area relates to that recommended by Hornby [6] in the creation of a hybrid system which incorporates the ALPS technique with a diversity preservation technique. Although Hornby recommended genotypic diversity, a system based on behavioural diversity would be well suited to the application of portfolio optimisation. See for example [21].

\section{ACKNOWLEDGMENT}

The authors thank Dr Gerard Vila, Wei Yan, and Prospect Wealth Management for suggestions and discussions, and Reuters for access to financial data. We also thank the anonymous referees for their helpful comments.

\section{REFERENCES}

[1] M. A. H. Dempster and C. M. Jones A Real Time Adaptive Trading System Using Genetic Programming Quantitative Finance 1:397-413, Institute of Physics Publishing, 2001.

[2] A. Ekart and S. Z. Nemeth Maintaining the Diversity of Genetic Programs Proceedings of EuroGP 2002, LNCS 2278, pp.162-171, Springer-Verlag, 2002.

[3] E. F. Fama Efficient Capital Markets: A Review of Theory and Empirical Work Journal of Finance, American Finance Association, 25:383-417, May 1970

[4] B. Graham The Intelligent Investor - Revised Fourth Edition Collins Business Essentials, 1973.

[5] C. Grosan and A. Abraham Stock Market Modeling Using Geneting Programming Ensembles Genetic Systems Programming: Theory and Experiences, Studies in Computational Intelligence, Vol 13, pp.133148, Springer, 2006

[6] G. S. Hornby ALPS: The Age-Layered Structure for Reducing the Problem of Premature Convergence GECCO 2006: Proceedings of the 8th annual conference on Genetic and Evolutionary Computation, Vol. 1, pp.815-822, ACM Press, 8-12 July 2006.

[7] J. J. Hu and E. D. Goodman The Hierarchical Fair Competition (HFC) Model for Parallel Evolutionary Algorithms Proceedings of the Congress on Evolutionary Computation 2002, pp. 49-54, IEEE Press, 2002

[8] H. Iba and T. Sasaki Using Genetic Programming to Predict Financial Data Proceedings of the Congress on Evolutionary Computation, Vol 1, pp.244-251, IEEE Press, 6-9 July 1999

[9] M. A. Kaboudan Genetic Programming Prediction of Stock Prices Computational Economica, 16:207-236, Kluwer Academic Publishers, 2000 .

[10] V.Karunamurthy, A Genetic Programming Approach to the Dynamic Portfolio Rebalancing Problem, Genetic Algorithms and Genetic Programming at Stanford 2003, pp. 100-108, Stanford Bookstore, 2003

[11] M. G. Kendall The Analysis of Economic Time-Series, Part I. Prices Journal of the Royal Statistical Society, 96:11-25, 1953.

[12] A. W. Lo and A. C. MacKinlay A Non-Random Walk Down Wall Street. Princeton University Press, 2001.
[13] B. G. Malkiel A Random Walk Down Wall Street W. W. Norton \& Company, 1973.

[14] W. F. Sharpe. The sharpe ratio. J. Portfolio Management, 21:49-58, 1994.

[15] N. Svangard and P. Nordin Generating Investment Policies for Nonlinear Portfolio Optimization with Genetic Programming, Proceedings of the Sixth International Conference on Computational Intelligence and Natural Computing, 2003

[16] N. Svangard, P. Nordin and S. Lloyd, Using genetic programming with negative parsimony pressure on exons for portfolio optimization, Proceedings of the 2003 Congress on Evolutionary Computation CEC2003, pp. 1014-1017, IEEE Press, 2003.

[17] L. Tang, M. Li and J. Zhang Multipopulation Genetic Programming For Forecasting Crop Pests IEEE Int. Conf. Neural Networks and Signal Processings 2003, pp.554-557, December 14-17 2003.

[18] G. Vedarajan, L. C. Chan and D. Goldberg, Investment Portfolio Optimization using Genetic Algorithms, Late Breaking Papers at the 1997 Genetic Programming Conference, pp. 255-263, Stanford University, 1997

[19] L. Wagman, Stock Portfolio Evaluation: An Application of GeneticProgramming-Based Technical Analysis, Genetic Algorithms and Genetic Programming at Stanford 2003, pp. 213-220, Stanford Bookstore, 2003

[20] J. C. Werner and T. C. Fogarty, Genetic control applied to asset managements, Genetic Programming, Proceedings of the 5th European Conference, EuroGP 2002, LNCS 2278:192-201, Springer-Verlag, 2002.

[21] W. Yan and C. D. Clack Behavioural GP Diversity for Dynamic Enviroments: an application in hedge fund investment GECCO 2006: Proceedings of the 8th annual conference on Genetic and Evolutionary Computation, Vol. 1, pp.1817-1824, ACM Press, 8-12 July 2006. 